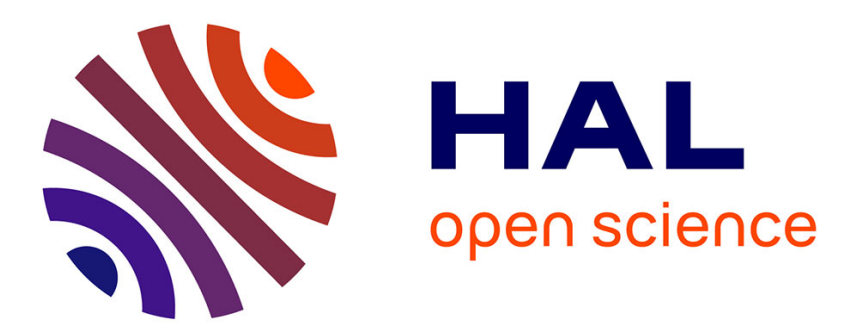

\title{
MOLECULAR POTENTIALS FOR SYSTEMS WITH ONE OR TWO ACTIVE ELECTRONS
}

\author{
F. Masnou-Seeuws
}

\section{To cite this version:}

F. Masnou-Seeuws. MOLECULAR POTENTIALS FOR SYSTEMS WITH ONE OR TWO ACTIVE ELECTRONS. Journal de Physique Colloques, 1985, 46 (C1), pp.C1-43-C1-59. 10.1051/jphyscol:1985105 . jpa-00224474

\section{HAL Id: jpa-00224474 https://hal.science/jpa-00224474}

Submitted on 1 Jan 1985

HAL is a multi-disciplinary open access archive for the deposit and dissemination of scientific research documents, whether they are published or not. The documents may come from teaching and research institutions in France or abroad, or from public or private research centers.
L'archive ouverte pluridisciplinaire HAL, est destinée au dépôt et à la diffusion de documents scientifiques de niveau recherche, publiés ou non, émanant des établissements d'enseignement et de recherche français ou étrangers, des laboratoires publics ou privés. 


\title{
MOLECULAR POTENTIALS FOR SYSTEMS WITH ONE OR TWO ACTIVE ELECTRONS
}

\author{
F. Masnou-Seeuws \\ Laboratoire des Colzisions Atomiques et Molécuzaires*, Bât. 351 , \\ Université Paris-Sud, 91405 Orsay Cedex, France
}

Résumé - Grâce aux méthodes de pseudo-potentiel et de potentiel modèle, des résultats très précis ont été obtenus pour les propriétés moléculaires des systèmes à un électron actif comme les couples alcal in-gaz rare. Pour traiter les dimères alcalins et leurs cations, il est important de décrire avec précision les effets de polarisation de cœur.

Abstract - Owing to model potential and pseudo-potential methods, very accurate results have been obtained for the molecular properties of systems with one active electron such as alkal i rare-gas couples. In order to treat the alkali dimers and their cations, it is important to perform an accurate description of core polarisation effects.

\section{I - INTRODUCTION}

Owing to laser techniques, much experimental information is now available concerning the ground and excited states of molecules and the dynamical or optical couplings between those states. In many cases, the molecular energies are known with an accuracy better than $1 \mathrm{~cm}^{-1}$. Such a situation is a challenge for theoreticians.

For many systems, the standard methods of quantum chemistry, using extensive configuration interaction, have succeeded in predicting accurate potential curves. However, it is generally difficult to reach an accuracy comparable to the experimental one. In the particular case of systems with one or two active electrons, model potential and pseudo-potential calculations, in which the effect of the rapid core electrons on the motion of the slow outer(s) electron(s) is simulated by an effective potential fitted on accurate atomic data, sometimes provide an excellent accuracy.

Concerning alkali-rare gas systems, it is seven and five years since a $1 \mathrm{~cm}^{-1} \mathrm{accu}^{-}$ racy has first been obtained in the laser spectroscopy of the ground state of NaNe $/ 1 /$ and $\mathrm{NaAr} / 2 /$. In the same period, a similar theoretical accuracy has been reached by model potential calculations /3,4/ using the method developped by Valiron et al. /5\%.

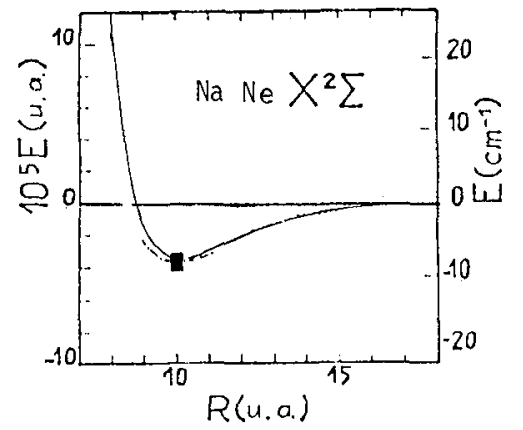

Fig. 1 - Model potential calculations for the ground state of $\mathrm{NaNe} / 3 /$ compared wi th laser spectroscopy determination /1/ (full 1 ine, theory; square and dotted line, experiment)

\footnotetext{
*Laboratoire associé $N^{\circ} 281$ au CNRS
} 
In such a method, the non local effects in the electron-rare gas interaction are represented by an explicit orthogonality condition to the outer orbitals of the rare gas. It has given good results for $\mathrm{Ne}$ and for $\mathrm{Ar} / 6 /$ perturbers. It might not be sufficiently accurate for $p$ wave scattering on an helium perturber. Recent calculations of Pascale /7/ using an 1-dependent pseudo-potential for the e-helium interaction have improved the agreement with experiment (see Fig. 2).

Fig. 2 - Normalised emission spectrum of the $\mathrm{Na} 3 \mathrm{p}$ state in the presence of He at $400 \mathrm{~K}$. Dashed 7 ine, experimental data of York et al $/ 8 /$, solid line calculations of Pascale $/ 7 /$, dash-dotted 1 ine results of Hanssen et al / 4 using the method described in $/ 5 /$. (from Ref. /7/)

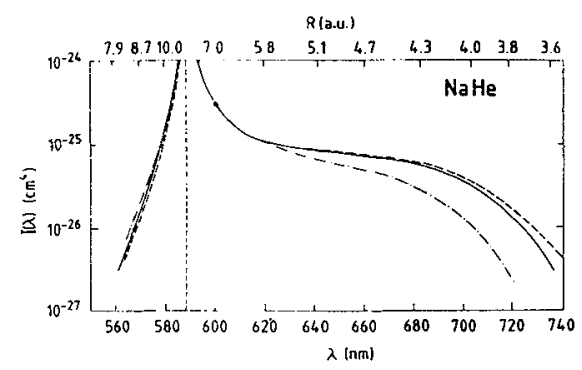

For the excited states, the accuracy depends upon the estimation of the core-core interaction at small internuclear distances, as we shall discuss later on.

During the last three years, the accuracy in the determination of the well in the ground state of the alkali dimer cations has reached a fraction of a wave number, due to extensive studies of the Rydberg states of the alkali dimers using twophoton techniques (see Ref. / 9/ to /16/). Such results have stimulated an intense theoretical activity in the field, and the importance of core polarisation effects for the obtention of high quality results has been stressed $17 / / 18 /$.

During the same period, the laser spectroscopy of the ground state and of the first excited states of the alkali dimers has reached an accuracy of a few wave numbers (see $/ 19 /, / 20 /$ and references therein). The most recent determination for the depth $\mathrm{D}_{\mathrm{e}}$ of the ground state of $\mathrm{Li}_{2} / 21 /$ and $\mathrm{Na} 2 / 22 /$ is $8516.9+0.4 \mathrm{~cm}^{-1}$ and $6022.6+1 \mathrm{~cm}^{-1}$ respectively. The theoretical results are on the point of reaching the experimental accuracy: Jeung /23/ obtains a well depth of $6020 \mathrm{~cm}^{-1}$ for the $\mathrm{Na}_{2}$ ground state, while konowalow and Fish /24/ estimate $D_{e}=8501 \mathrm{~cm}^{-1}$ for Li2. In both case, the inclusion of core polarisation effects is crucial, and the two treatments are intermediate between $\alpha b$ initio and effective potential methods.

My a im is to present some of the results obtained recently, and to discuss how in the next future very accurate results could be obtained for the excited states.

\section{2 - EFFECTIVE POTENTIALS FOR THE ALKALI ATOMS : APPLICATION TO ALKALI-RARE GAS SYSTEMS}

The basic idea of model potential and pseudo-potential methods is to treat an alkali-rare gas system by solving a one electron Schrödinger equation in order to describe the motion of the active electron in the field of the two cores $A$ and $B$ at a distance $R$

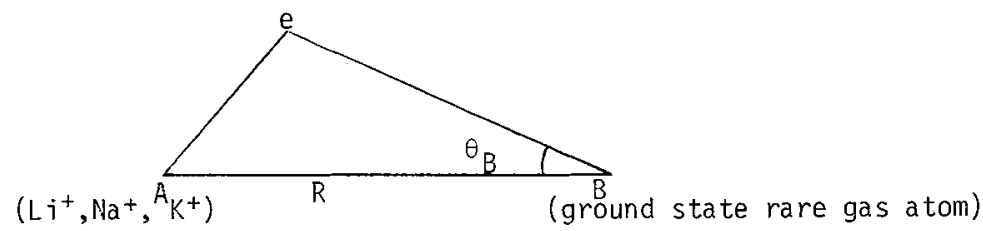

Fig. 3 - Coordinate system 
We therefore must solve the equation :

$$
H \Upsilon_{i}^{\lambda}=\left[T+V_{A}\left(r_{A}\right)+V_{B}\left(r_{B}\right)+V_{3}\left(\underset{\sim}{R}, r_{A}, r_{B}\right)\right] \Upsilon_{i}^{\lambda}=E_{i}^{|\lambda|} \Upsilon_{i}^{\lambda}
$$

In (1) $T$ is the kinetic energy, $V_{A}\left(r_{A}\right)$ and $V_{B}\left(r_{B}\right)$ are the effective potentials for the interaction of the electron with the cores $A$ and $B$ respectively, $V_{3}$ is a threebody tensorial term which depends upon the angle $\theta_{B}$ and comes from the addition of the two electric dipoles induced in $B$ by $A$ and $e^{-}$respectively.

\section{1. - Definition of $V_{A}\left(r_{A}\right)$, effective potential for the alkali atoms}

In most cases, $V_{A}\left(r_{A}\right)$ is a parametric potential fitted to properties of the isolated e-A system. The atomic orbitals of the alkali atom are obtained by solving (2)

$$
\left[T+V_{A}\left(r_{A}\right)\right] \Phi_{i}^{n}\left(r_{A}\right)=E_{i}^{a t} \phi_{i}^{m n}\left(\underline{r}_{A}\right)
$$

The usual procedure consists in fitting the parameters in $V_{A}$ so that the calculated energies $E_{i}^{a t}$ are as close as possible from the experimental ones. In a model potential treatment $/ 25 / V_{A}\left(r_{A}\right)$ is attractive and contains virtual core orbitals besides the valence orbitals. The atomic functions $\phi^{m}$ therefore possess the correct number of nodes. In a pseudo-potential treatment $/ 26 /, V_{A}\left(r_{A}\right)$ is repulsive in the core region, leading to nodeless pseudo-orbitals at small ra. This is illustrated on Fig. 4

MODEL POTENTIAL

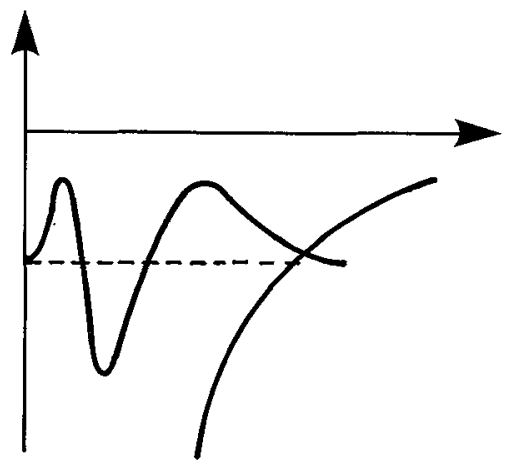

PSEUDO POTENTIAL.

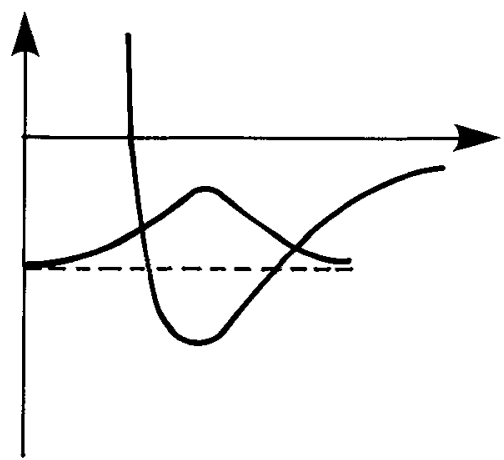

Fig. 4 - Schematic representation of the Na 3 s orbital in model and in pseudopotential calculations.

The first question one could ask is then : which is the true potential ? We shall show here below that it is a wrong question.

Indeed, the definition of an effective potential comes from an adiabatic separation between the motion of the rapid (core) electrons and of the slow (valence) electrons. Calling $r_{1} \ldots r_{Z 1}$ the positions of the core electrons, and $r_{A}$ the position of the valence electron, we may write $/ 27,28 /$ the total wavefunction for the ground state of the atom $A$

$$
x_{0}\left(r_{1} \ldots . . r_{z-1} \mid r_{A}\right) \phi\left(r_{A}\right)
$$

In (3) $x_{0}$ is the wavefunction for the motion of the core electrons for a fixed position $r_{A}$ of the valence electron. The adiabatic equation :

$$
\left[T+W\left(r_{A}\right) \cdot \frac{1}{r_{A}}\right] \phi\left(r_{A}\right)=\varepsilon \phi\left(r_{A}\right)
$$


defines the model potential $W\left(r_{A}\right)-1 / r_{A}$ and describes the motion of the slow electron in the mean potential of the core. The physical ideas involved are therefore close to the Born-oppenheimer separation in molecular physics. The non adiabatic correction :

$$
J\left(r_{A}\right)=-\frac{1}{2} \iint x_{0}\left(r_{1} \ldots r_{2-1} \mid r_{A}\right) \overrightarrow{g r a d}_{r_{A}}^{2}\left(x_{0}\left(r_{1} \ldots r_{2-1} \mid r_{A}\right)\right) d \tau
$$

is small, and 7 imited to the core region. It has been computed through a variational procedure in the case of the 7 ithium atom $/ 27 /$.

The best effective potential may therefore be defined so that outside the core region the wavefunctions solutions of (2) are as close as possible from the "exact" wavefunctions. The physical ideas are therefore very similar to quantum defect theory. The short range potential is an empirical tool for making sure that a large number of excited state wavefunctions have a correct quantum defect outside the core. As in quantum defect theory, $V_{A}\left(r_{A}\right)$ is independent of the energy of the level considered, but is usually 1-dependent.

One way of obtaining a good valence wavefunction is to vary the parameters in the potential so that the calculated atomic energies in (2) are as close as possible from the experimental energies. Bottcher and Dalgarno $/ 29 /$, using a $2^{\text {nd }}$ order perturbation theory, have shown that core polarisation effects should be included in the effective potential. This can be done by introducing in the potential an effective core polarisation operator :

$$
V_{p o l}\left(r_{A}\right)=-\frac{\alpha_{d}^{A}}{2 r_{A}^{4}} f\left(\frac{r_{A}}{r_{d}}\right)-\frac{\alpha_{q}^{A}-6 \beta_{d}^{A}}{2 r_{A}^{6}} g\left(\frac{r_{A}}{r_{q}}\right)
$$

In (6), $\alpha_{d}^{A}$ and $\alpha_{q}^{A}$ are respectively the dipole and quadrupole static polarisabilities of the core, $\beta_{d}^{A}$ being the dynamic dipole polarisability - $f$ and $g$ are cut-off functions necessary to avojd the divergence in the core region, $r_{d}$ and $r_{g}$ being cut -off parameters typical of the core dimensions. Two comments should be added:

1) The core electrons being not explicitzy included in the treatment, the properties of the core such as its polarisation appear as an effective operator.

2) The behaviour of the system during the short period of time when the electron enters the core region is treated as a "black box". The parameters $r_{d}$ and $r_{q}$ must then be fitied on experimental data.

In fact, the calculated energies are not very sensitive to the choice of such parameters $/ 30 \%$. Various authors using a large variety of analytical formulas for the potential, have succeeded in reproducing the optical spectrum of the alkal $i$ atoms with a good accuracy $/ 31 /$.

In contrast, the energy variation of the photoionisation cross-sections of the alkali atoms is very sensitive to the choice of the cut -off parameters $/ 18 /$. A possible improvement in the determination of the effective potential would consist in fitting directly the experimental photoionisation cross-sections. The influence of such a choice will be discussed later on.

\section{2 - Definition of $V_{B}\left(r_{B}\right)$ and of $V_{3}$}

$V_{B}\left(r_{B}\right)$ represents the interaction of a free electron with a neutral rare gas and is usually fitteci on low energy electron-rare gas scattering data ; $32 /$ which at present time are known very accurately. We may use the expression :

$$
V_{B}\left(r_{B}\right)=V_{S-}\left(r_{B}\right)-\left(\alpha_{d}^{B} / 2 r_{B}^{4}\right) h_{1}\left(r_{B} / \rho_{d}\right)-\left(\alpha_{q}^{\prime B} / 2 r_{B}^{6}\right) h_{2}\left(r_{B} / p_{q}\right)
$$

in which $v_{S r}$ is a short range term (attractive on repulsive, see $\hat{\beta} 2.1$ ), $\alpha$ and 
$\alpha_{q}^{\prime} B=\alpha_{q}^{B}-6 \beta_{d}^{B}$ are the polarisajilities of the rare gas, $\rho_{d}$ and $\rho_{q}$ cuti-off radi $i$

$d$ typical of the dimensions of the core $B$. The computed phase-shifts are usually sensitive to the choice of such parameters. Besices, as was said in the introcuction, the short range term $V_{S r}\left(r_{B}\right)$ must be non local $(5,7)$. The tensorial term: $V_{3}$, anc hence the molecular energies, also depend upon the choice of the cut-off parameters :

$$
V_{3}\left(\underset{2}{R}, r_{B}\right)=\frac{\alpha_{d}^{B}}{r_{B}^{2} R^{2}}\left(h_{1}\left(\frac{r_{B}}{\rho_{d}}\right)\right)^{1 / 2} \cos \theta_{B}+\frac{\alpha_{q}^{B}}{r_{B}^{3} R^{3}}\left(h_{2}\left(\frac{r_{B}}{\rho_{q}}\right)\right)^{1 / 2} P_{2}\left(\cos \theta_{B}\right)
$$

$\theta_{B}$ (see fig. 1) is the angle $\left(R, K_{B}\right.$ ). Peach /33/ has shown that the cut-off functions in (8) should be the square root of those involved in (7).

It is important to remark that $V_{B}\left(r_{B}\right)$-as well as the sum $v_{B}\left(r_{B}\right)+V_{3}\left(R, r_{B}\right)$ - is a short range termi compared to the $1 / r_{A}$ behaviour of $V_{A}\left(r_{A}\right)$. The rare gas $Z$ may then be considered as introducing an additionnal $R$ dependent quantum defect on the alkali atomic wavefunctions. On Fig. 5 and 6 we have represented both the atomic wavefunction $\phi m_{0}=0\left(r_{A}\right)$ solution of $(6)$ and the molecular wavefunction $\psi d=0\left(r_{A}, R\right)$ solution of $(1)$ oblained in model potential calculations for the NaHe and 6 Rave systems.

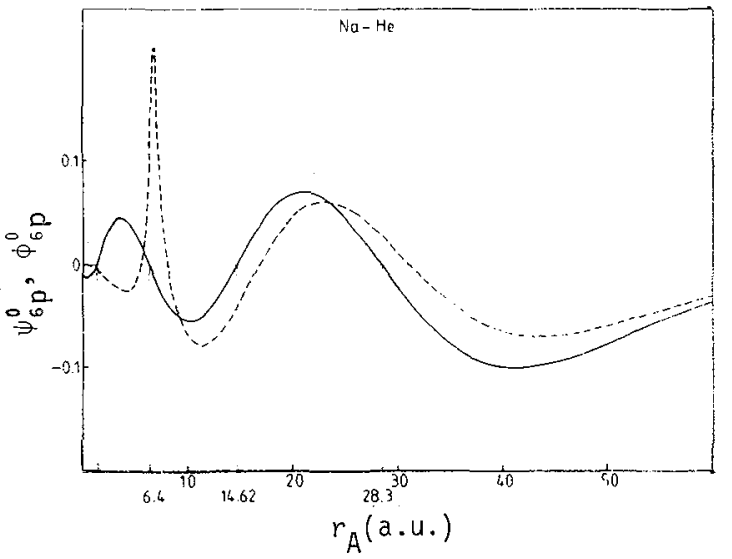

Fig. 5 (fror. Ref. 34) - Solid 1 ine : sodium atomic wavefunction $\phi_{6 p}^{m=0}\left(r_{A}\right)$ as a function of the distance $r_{A}$ on the quantization axis. Dashed Tine : $6 p$ molecular wavefunction $\psi_{6 p}^{\lambda}=0\left(r_{A}, R=6.75 r_{A} / r_{A}\right)$ in case of an hel ium perturber 1ocated at 6.75 a.u. of the sodium center.

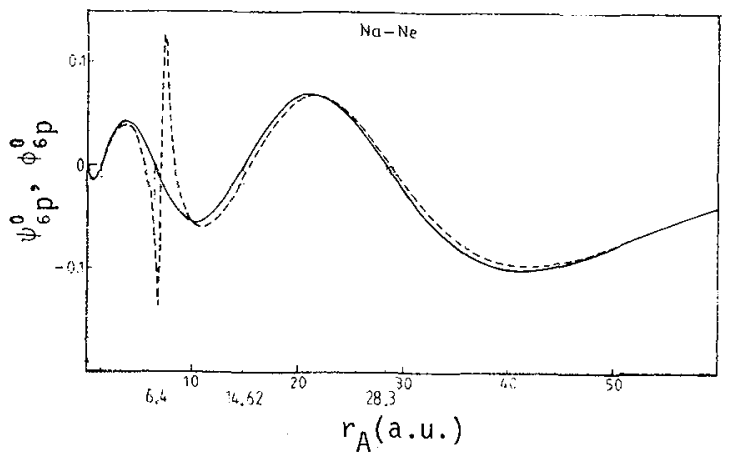

Fig. 6 - Same as Fig. 5 in case of a Ne perturber. 
It is clear from Fig. 5 and 6 that :

a) the molecular wavefunction is very similar to the atomic one, except in the rare gas region where it is strongly modified due to the orthogonality constraint with the occupied orbitals.

b) the basis set used in model potential calculations must be flexible enough to deal correctly with the modification of the alkali wavefunciton in the region of the rare gas core

\section{3 - Results for the ionisation energies : molecular quantum defects}

The eigenenergy $E_{i}|\lambda|$ computed in $(1)$ is the ionisation energy, i.e. the energy of an electron in the field of the two cores $A^{+}$and $B$ at distance $R$ for a molecular state of symmetry $\lambda$ correlated to an atomic $|n \ell m\rangle=|i\rangle$ state.

From the predictions of the Fermi model /35/ and of the asymptotic methods /36/, it has long been known that the difference between the ionisation energy $E|\lambda|(R)$ of a molecular Rydberg state of an alkali rare gas system and the energy Eat of the corresponding atomic state can be scaled by:

$$
E_{i}^{|\lambda|}(R)-E_{i}^{a t}=a_{0}(k(\underset{\sim}{R}))\left|\phi_{i}^{m=\lambda}(R)\right|^{2}+a_{1}(K(R))\left|\underset{\sim}{\sim} \phi_{i}^{m=\lambda}(\underset{\sim}{R})\right|^{2}+\ldots
$$

where $\left|\phi_{i}^{m}(R)\right|^{2}$ is the probability density of the atomic wavefunction at the rare gas center, $K(R)$ the local de Broglie wavelength associated to it. The quantities $a_{0}(k)$ and $a_{1}(k)$ are respectively proportionnal to the $s$ and $p$ wave phase shifts for the elastic scattering of an electron with a wavenumber $k$ by the rare gas $B$. Such a formula is valid in a region where $K(R)$ is a slowly varying function of $R$. We have represented on Fig. 7 the ionisation energies of the $\sigma 4 p$ and $\pi 4 p$ states of the KHe, KNe and KAr systems.

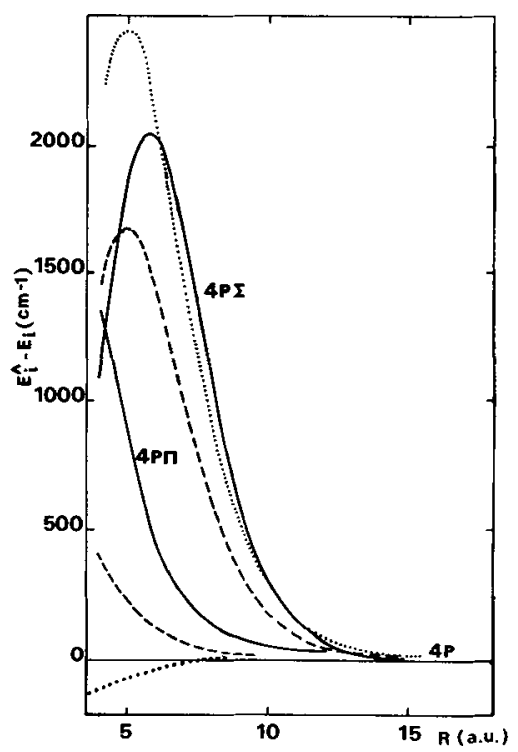

Fig. 7 - Ionisation energies (see tex $t$ ) of the $04 p$ and $\pi^{4} p$ states of KHe (dotted line), KNe (broken line) and $\operatorname{KAr}$ (full line) as a function of the internuclear distance R. $/ 6 /$. 
The maxima in the $\Sigma$ states correspond to maxima in the atomic wavefunction so that the rare gas can be considered as "measuring" the atomic probability density at a given point. The $\pi$ ionisation energies are proportionnal to the square of the gradient of the atomic wavefunction, which is a severe check on the accuracy of this wavefunction.

The representation of the rare gas $B$ as an additionnal $R$-dependent quantum defect is illustrated on Fig. 8, where we have represented as a function of the internuclear distance $R$ the molecular quantum defects $\delta ?$, with

$$
E_{i}^{|\lambda|}(R)=\frac{-R_{0}}{\left(m_{i}-\delta_{\lambda}^{\top}(R)\right)^{2}}
$$

In (10) $R$ is the Rydberg constant and $n_{j}$ the principal quantum number at infinity. We consider three atemic quantum defects, $\delta^{S}, \delta^{P}$, and $\delta \cong 0$ for $\ell \geqslant 2$.
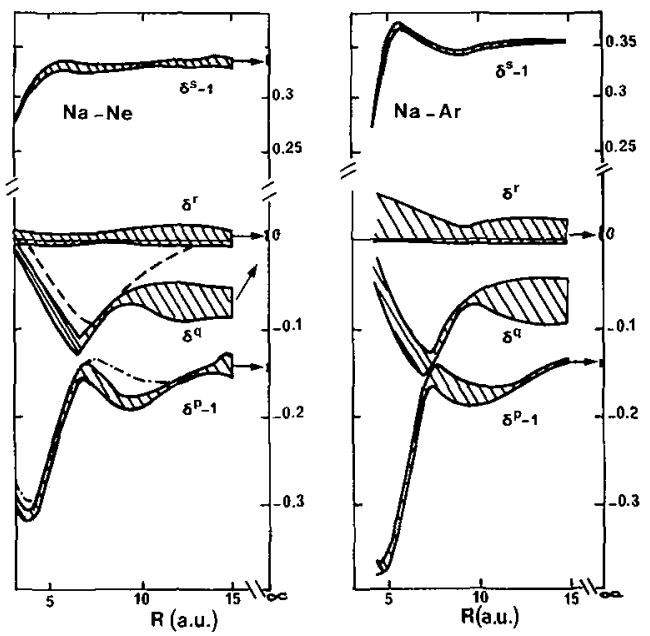

Fig. 8 - Molecular quantum defects for the 4po to 9so states of the NaNe and NaAr molecules. The quantum defects curves $\delta^{j}$ (see text) are contained in the shadowed areas $/ 6 /, / 37 /$. The $\delta 9$ and $\delta^{r}$ curves are correlated to atomic states with $\ell \geqslant 2$.

It is clear from Fig. 8 that the molecular quantum defects are fairly $n$-independent. For the states correlated to atomic states with $\ell \geqslant 2$, the quantum defect curve splits into two curves, one of which exhibits an avoided crossing with the $\left(\delta^{P}-1\right)$ curve. It has then been possible to predict collisionally induced transfers between the $\mathrm{Na}$ $((n+1) p)$ and $\mathrm{Na}$ nd states. The experimental measurements concerning such transfers /38/ are in excellent agreement with theoretical calculations /34/ in which the computed radial and rotational coupling matrix elements are introduced in the collision problem.

In conclusion, one may say that the accuracy of the ionisation energies is due to the quality of the atomic wavefunctions and to the precision of the electron rare gas elastic phase shifts obtained with the effective potentials. Such an accuracy is possible because the motion of the core electrons is sufficiently rapid to allow the introduction of effective potentials. 


\section{4 - Results for the molecular energy : problem of the core-core term}

The energy $\mathcal{\varepsilon}\left|\lambda_{i}\right|$ of a molecular $\mid \lambda i>$ state is obtained by adding to the ionisation energy $E] \lambda \mid$ the core-core energy $V_{A B}+(R)$, i.e. the energy of the ground state of the $A B^{+}$holecular ion for an internuclear distance $R$.

$$
\varepsilon_{i}^{|\lambda|}(R)=E_{i}^{|\lambda|}(R)+V_{A B^{+}}(R)
$$

$V_{A B}+(R)$ may be expressea as the sum of a polarisation term and of a short range repulsive term due to the overlap of the asymptotic part of the two core wavefunctions (Obviously, the method is no more valid when the atomic clouds are modified by the interaction of the two cores)

$$
V_{A B^{+}}(R)=V_{0}(R)-\left(\alpha_{d}^{B} / 2 R^{4}\right)-\left(\alpha_{q}^{B} / 2 R^{6}\right)
$$

If the polarisabilities $\alpha_{d}^{B}$ and $\alpha_{\mathrm{q}}^{B}$ of the core $B$ are well known, the quantity $V_{o}(R)$ -which is generally fitted to ion -atom scattering data or to mobility measurementsis not known very accurately, so that the $1 \mathrm{~cm}^{-1}$ accuracy available for the ionisation energies is obtained for the molecular energies only at large distances where $V_{A B}+$ (R) is limited to the polarisation terms. We have shown on Fig. 1 the excellent agreement which exists between theory and experiment concerning the well in the ground state of the NaNe system at $10 \mathrm{a} . \mathrm{u}$. In contrast (see (9)) the well of the first excited $I$ state is mainly determined by $V_{A B}+(R)$ and therefore depends markedly upon the estimation of $V_{0}(R)$. This is illustrated on Fig. 9 where we have represented the $\pi 4 p$ curve of $K A r$ for two different choices of $V_{A B+}(R)$

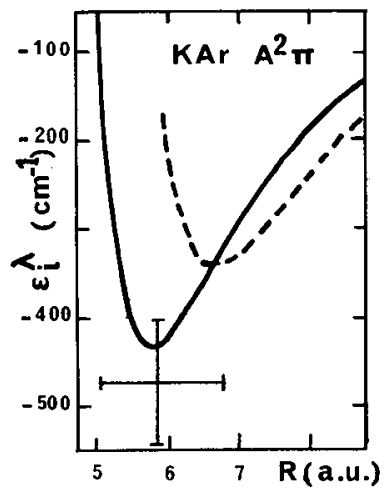

Fig. 9 - Molecular energy curve $\mathcal{E} 1$ (see formula (11) in text) of the $A^{2}$ II state of $\mathrm{KAr} / 6 /$, obtained through model pbtential calculations with two choices for the core-core term. Solid line: $\left\{{ }_{O}(R)\right.$ has been taken from Ref. /39/; dashed 1 ine : $\mathrm{V}_{\mathrm{AB}^{+}}(\mathrm{R})$ is taken from Ref. $/ 40 /$; Cross : experimental determination of Dïren and Tischer $/ 41 /$ for $\frac{1}{4 p}(R)$.

However, the core-core term is common to all molecular curves. It should be yajuable, especiaily when accurate experimental information is available for several $\varepsilon|\lambda|$ curves from collision or spectroscopy experiments, to fit $V_{A B}(R)$ directly on experimental results for the $A B$ system. In the work of Diren's group /42/ parameters in $V_{B}\left(r_{B}\right), V_{3}\left(R, r_{B}\right)$ and $V_{A B}(R)$ have been fitted so that accurate experimental scattering cross-sections can be reproduced from collision calculations involving the ellal $(R)$ curves. It should be very interesting to use a similar procedure with the curves $E_{i}(R)$ computed from (1) while $V_{A B}+(R)$ depends upon adjustable parameters. 
3 - GeNeralisation tO OPEN SHELl CORES : CALCULATIONS FOR HeHe*, $\mathrm{HeNe}^{*}$, NeNe*

The effective potential method has been general ised to open shell cores $/ 46,33,48 /$. For such systems, several states of the $A B+$ molecular ion are involved in the calculation, and humps in the excited curves ( $1 \mathrm{~s}_{5} \mathrm{O}_{-}^{-}$and $1_{u}$ of $\mathrm{Ne}_{2}$ ) depend markedly upon the estimation of the core-core interaction $148 /$. The accurate estimation of the $A B^{+}$curves is an open problem at present time.

Fig. 9bis. Hunip in the $0_{\bar{u}}^{-} 1 s_{5}$ potentia? curve of $\mathrm{Ne}^{*}-\mathrm{Ne}$.

Solid line, dotted line : model potential calculations $i 48 /$ for two different estimations of the core-core term Dashed 1 ine : fitted $/ 47 /$ to scattering experiments

\section{4 - STUDY OF THE ALKALI MOLECULAR IONS}

\section{1 - Molecular quantum defects}

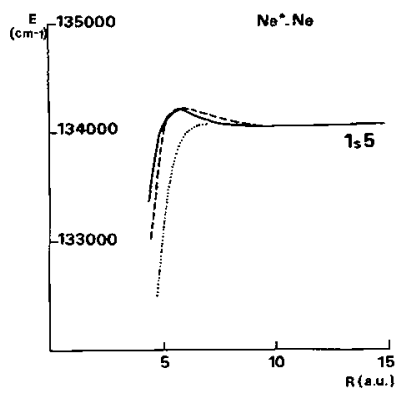

In contrast, when we turn to alkali molecular ions such as $\mathrm{Li}_{2}^{+}, \mathrm{Na}_{2}^{+}, \mathrm{K}_{2}^{+}$, the problem of the core-core term is less crucial, due to the predominance of the $T / R$ term. Nevertheless, the physical situation differs markedly from the preceeding one, as the electron now moves in the field of two charged cores. In the alkali rare gas problem, we have shown that an atomic wavefunction is modified by a R-dependent quantum defect. In the alkal i molecular ion problem, we may consider that the short range non coulombic term in the two potentials $V_{A}$ and $V_{B}$ introduces two quantumi defects on the $\mathrm{H}_{2}^{+}$wavefunctions. Such a situation is $i 1$ lustrated on Fig. 10 where we have represented the R-variation of the effective quantum number $n_{j}-\delta_{j}^{o}(R)$ for the $\Sigma$ states of the $\mathrm{Na}_{2}^{+}$ion:

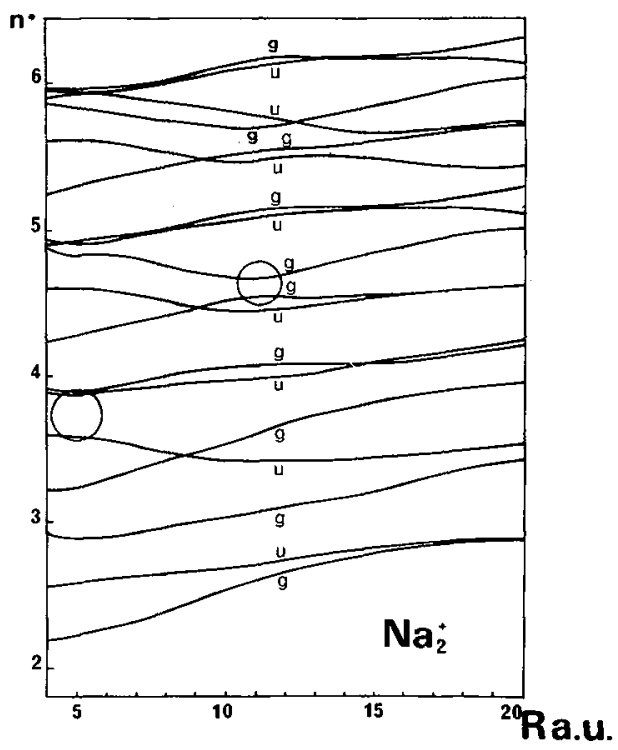

Fig. 10 - Variation of the effective quantum number $n_{i}-\delta_{0}^{i}(R)$ (see Eg (10)) as a function of the internuclear distance $R$ for the ground and excited $2 \Sigma$ states of the Nat molecular ion /18/ 
Avoided crossings are clearly visible, which would be permitted in the $\mathrm{H}_{2}^{+}$problem. In the $\mathrm{Na}_{2}^{+}$case, the potential departs from a pure coulombic potential in the core region, and the supersymetry of the $\mathrm{H}_{2}^{+}$problem is broken. It should be worthwile to perform on such systems experimental investigations similar to the study of Stark effect on alkali atoms.

\section{2 - Ground state of the alkali dimercations : core polarisation effects}

Model potential and pseudo potential calculations have been used successfully for many years in order to compute the properties of the ground and excited states of such systems $125,26 /$. It has long been known that the results are sensitive to the introduction of core polarisation effects. Ab initio calculations, taking account approximately of core polarisation, generally overestimate the bond length $/ 43 /$.

Recentvery accurate experimental determinations, obtained through two step ionisation techniques /10-16/ have stimulated new calculations Limiting the polarisation terms to the dipole terms, one obtains the molecular energies by solving the Schrödinger equation :

$\left[T+V_{A}\left(r_{A}\right)+V_{B}\left(r_{B}\right)+V_{3}\left(\underset{\sim}{R}, r_{A}, r_{B}\right)-\frac{\alpha_{d}^{A}}{R^{4}}+\frac{1}{R}\right] \Psi_{i}^{\lambda \lambda}\left(\underset{\sim}{R}, r_{A}, r_{B}\right)=\varepsilon_{i}^{\lambda} \Psi_{i}^{\lambda \lambda}\left(R, r_{A}, r_{B}\right)$

$V_{A}(r)$ is the potential defined in $\S 2.1$ and $\alpha A$ the polarisability of the two identical cores. The cross polarisation term is now deduced from (6)

$$
V_{3}\left(\underset{\sim}{R}, r_{A}, r_{B}\right)=\left(\alpha_{d}^{A} / R^{2}\right)\left\{r_{A}^{-2} \cos \left(R, r_{A}\right)\left[f\left(r_{A} / r_{d}\right)\right]^{1 / 2}+r_{B}^{-2} \cos \left(R, r_{B}\right)\left[f\left(r_{B} / r_{d}\right)\right]^{1 / 2}\right\}
$$

Because of the importance of the $V_{3}$ term, the results depend markedly upon the choice of the cut-off radius $r_{d}$. This is illustrated on Fig. 11:

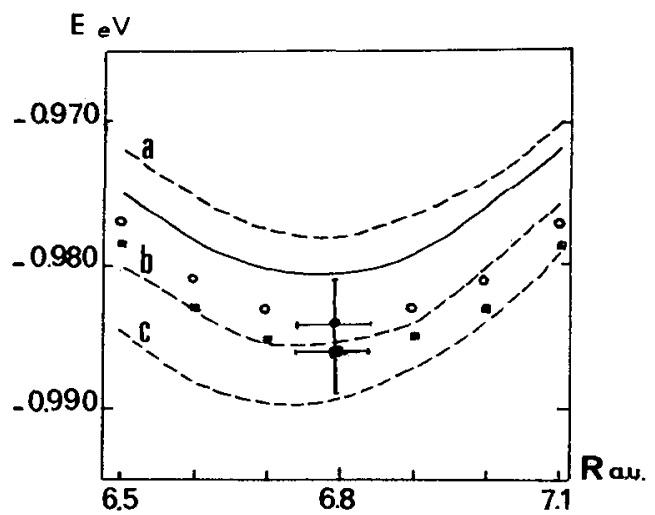

Fig. 11 - Potential curve for the ground state of $\mathrm{Na}_{2}^{+} / 18 /$ in the minimum region for various choices of the cut-off radius $r_{d}$. Dashed 1 ines : calculations using $(a) r_{d}=2$ (b) $r_{d}=3$ (c) $r_{d}=3.5$ a.u. Ful1 1 ine : calculations using Weisheit's value $r_{d}=2.35$ (see text). Circies : experimental results $/ 13 /$. Squares : experimental results $/ 14 /$.

The determination of the cut-off appears then as an important problem. The $V_{p o l}$ term in $V_{A}$ (see (6)) depending upon $r_{d}$, one possibility is to fit the cut-off radius so that the atomic energies eigenvalues of (2) are as close as possible from experiment. Such a procedure has been used in recent pseudopotential calculations $/ 17 /$. However, recent work using model potential calculations $/ 18 /$ has shown that the atomic 
energies are not very sensitive to the choice of $r_{d} / 44 /$, introducing some arbitrariness in the method. In contrast, however, the atomic dipole moments /28/ and the photoionisation cross sections /45/ are sensitive to $r_{d}$. The value $2.35 \mathrm{a} . u$. fitted by Weisheit /45/ in order to reproduce the minimum in the photoionisation cross-section leads to a satisfactory potential curve $1.18 /$ for the ground state of $\mathrm{Na}_{2}^{+}$(see Fig. 11). The results of $/ 17 /$ and $/ 18 /$ are compared to experiment on Table 1. In both cases, we have indicated in italics the values obtained when neglecting the cross polarisation term $V_{3}$ in the effective hamiltonian.

TABLE 1

Ground state properties of $\mathrm{Li}_{2}^{+} \mathrm{Na}_{2}^{+} \mathrm{K}_{2}^{+}$

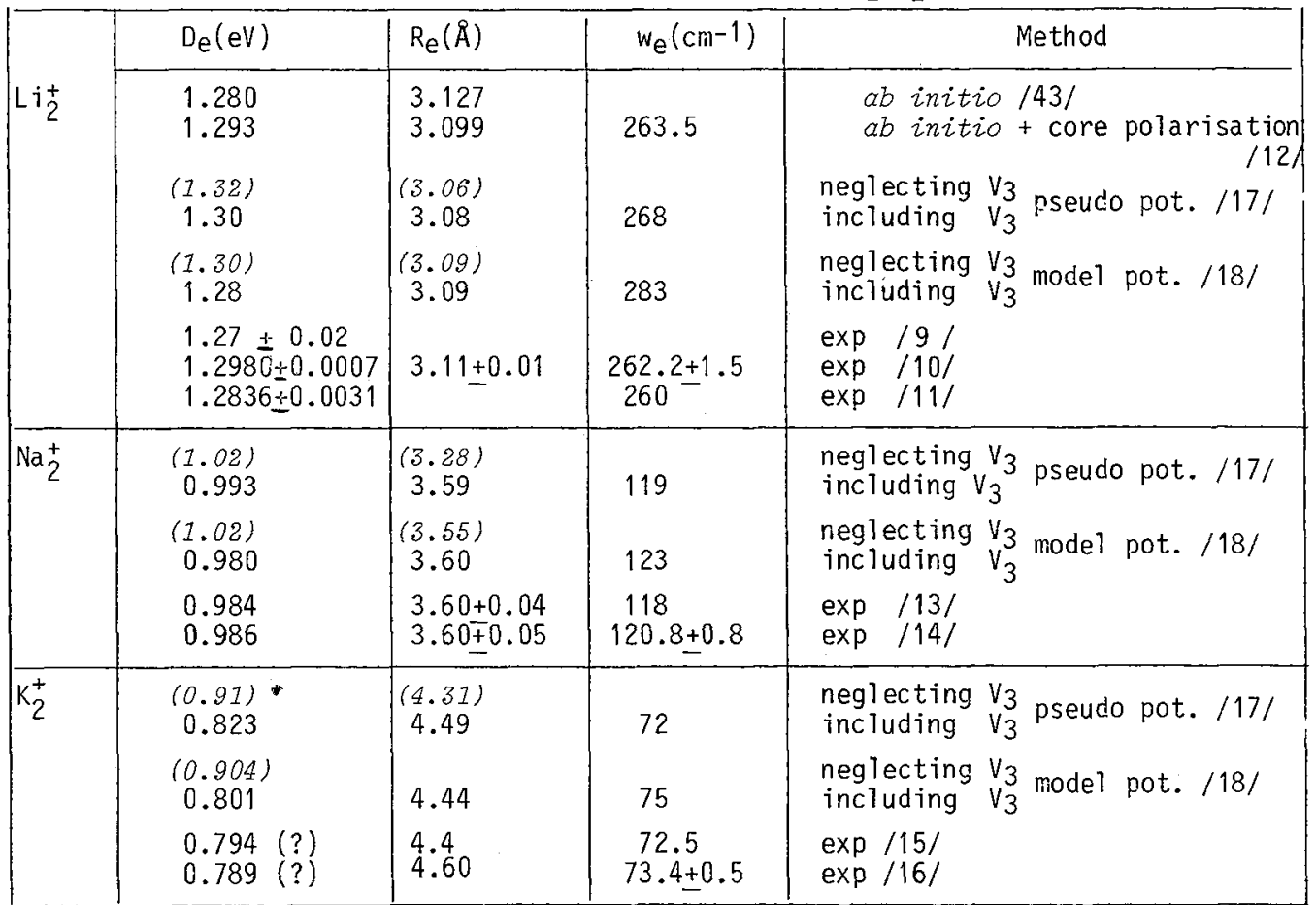

In the first two 1 ines of table 1 are reported $\alpha b$ initio results for the ground state of $\mathrm{L}^{\mathrm{i}} \mathrm{2}$. When core polarisation effects are neglected /43/ the bond length is overestimated. In contrast, ab initio calculations corrected for core polarisation $/ 127$ provide a bond length in better agreement with experiment.

The importance of the inclusion of the cross polarisation term $V_{3}$ is clear also from table 1 especially for $K_{2}^{+}$. When the active electron and the core $A$ (resp B) simultaneously polarise the core $B$ (resp (A)) the resulting force is the sum of the two

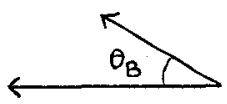
polarisation forces and depends upon $\theta_{B}$ (see Fig. 1). When we neglect the term $V_{3}$, we assume that the two forces are collinear so we overestimate the polarisation forces and hence the werl depth.

The agreement between theory and experiment is satisfactory when $v_{3}$ is included. In the case of the $K^{\frac{1}{2}}$ ion, the value of the experimental well depth depends upon the well depth in the ground state of the $K_{2}$ molecule, which is not known accurately a present. Nevertheless, the experimental accuracy is not yet reached, and further work including a more precise fitting of the cut-off radius $x^{\prime} d$, possibly on atomic photoionisation cross sections, is needed. 
5 - TREATMENT OF THE TWO ELECTRON PROBLEM : APPLICATION TO ALKALI DIMERS

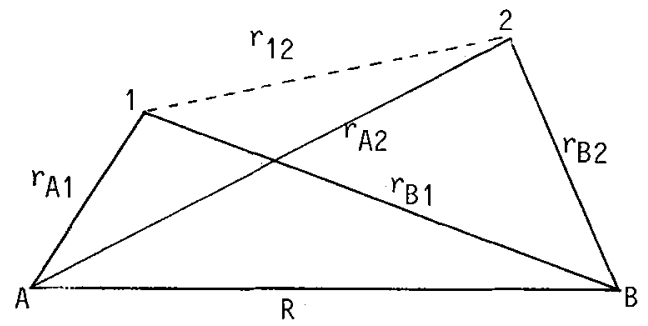

Fig. 12. - Coordinate system

In the case of two electrons moving in the field of two alkali cores $A$ and $B$, it is necessary to treat simultaneously

a) the interaction of the electrons with the two cores
b) the bielectronic interaction

It is beyond the scope of the present paper to review a 17 the theoretical work on that subject; an extensive bibliography is available for Li2 and Na2 /19,20/. It seems that four kinds of treatment are possible nowadays, according to the methods used to deal with the two problems in a) and b)

\section{1 - Effective operator method with configuration interaction}

The method described in $\$ 4$ for the molecular ions has been extended to two electron systems. The model potential treatment has been generalised by Dalgarno's group /25, 49/ while a pseudo-potential treatment of alkali dimers has been developped first by Bardsley and his coworkers $/ 26,50 /$, then by Valance et al, who treated the excited states $/ 51 /$. The total hamiltonian is written :

$$
H=h(1)+h(2)+V(1,2)-V_{A B}+(R)
$$

where $h(j)$ is the one electron operator defined in (13)

$$
h(j)=T+V_{A}\left(r_{A j}\right)+V_{B}\left(r_{B j}\right)+V_{B}\left(\underset{\sim}{R}, \underset{r_{A} i}{r_{i}} \underset{\sim}{r_{B}}\right)+V_{A B+}(R)
$$

and describes the motion of the active electron $i$ in the field of the two cores. Core polarisation effects are included and it its clear from the perturbative treatment of Bottcher and Dalgarno /29/ that all the one electron operators must be replaced by effective operators, depending of a cut-off radius typical of the core dimensions. For instance, the dipole moment is modified $/ 28 /$

$$
\Gamma_{A i} \rightarrow r_{A i}\left(1-\alpha_{d}^{A} r_{A i}^{-3}\left[f\left(r_{A i} / r_{d}\right)\right]^{1 / 2}\right)
$$

and the bielectronic interaction must. include a dielectric term $v_{\text {diel }}$

$$
V(1,2)=\frac{1}{r_{12}}-\frac{\alpha d \cos \left(r_{A 1}, r_{A 2}\right)}{r_{A 1}^{2} r_{A 2}^{2}}\left[f\left(\frac{r_{A 1}}{r_{d}}\right) f\left(\frac{r_{A 2}}{r_{d}}\right)\right]-\frac{\alpha_{d}^{B} \cos \left(r_{B 1}, r_{B 2}\right)}{r_{B 1}^{2} r_{B 2}^{2}}\left[f\left(\frac{r_{B 1}}{r_{d}}\right) f\left(\frac{r_{B 2}}{r_{d}}\right)\right]^{1 / 2}
$$

The dielectric correction, first introduced by Chisholm and 0pik $/ 53 /$, comes from the non additivity of the two potentials for the polarisation of one core by the two electrons simultaneously, and is a cross polarisation term as $V_{3}$. It has to be included in accurate calculations of the affinity of the alkali atom $/ 54 /$. The method is simple because the core electrons are not explicitly included : this can be done provided one may neglect the valence excitation energies compared to the core excitation energies $129 \%$. The results depend upon the choice of an arbitrary cut-off radius $r_{d}$, which has to be chosen unambiguously.

After solving the Schrödinger equation for the molecular ion problem :

$$
h(i) \Psi_{a}^{\lambda_{a l}}=E_{a}^{\lambda_{a}} \Psi_{a}^{\lambda_{a}}\left(\underset{\sim}{R}, \Gamma_{A i}, \Gamma_{B i}\right)
$$


one performs a standard configuration interaction treatment by expanding the total wavefunction on antisymetrized products of monoelectronic orbitals solutions of (13)

$$
F(1,2)=\sum_{a b} \text { of } \Psi_{a}^{\lambda_{a}}(1) \Psi_{b}^{\lambda_{b}}(2)
$$

and computing the matrix elements of $\vartheta(1,2)$.

Such a method has given good results, but in many existing treatments either the cross polarisation term $V_{3}$ or the dielectric term Viet is neglected.

\section{2 - Effective operator method using correlated orbitals}

Recently methods have been proposed which treat the one electron problem as in the preceeding case but improve the calculation of the two electron correlation. Preuss' group has developped a sophisticated treatment of core polarisation effects, including $V_{3}$ and $V_{\text {diel }} / 52 /$. A non local pseudopotential is fitted to the experimental valence energy of the isolated atoms, and the two electron problem is solved in the framework of the density functional formalism, the charge density of valence electrons with spin + and - respectively being not necessarily identical. The interest of such a method is that it has easily been generalised to alkali clusters.

Another approach /55/ treats the one electron problem as in the preceeding case, but improves the calculation of the two electron correlation owing to a generalisation of Pluvinage's treatment for the hel ium atom $/ 56,57 /$. Equation (20) is modified to :

$$
F_{p}(1,2)=\sum_{a b} \text { of } \Psi_{a}^{\lambda_{a}}(1) \Psi_{b}^{\lambda_{b}}(2) u_{k}^{\lambda_{k}}\left(r_{12}\right)
$$

in which $u_{k}^{\lambda} \quad\left(r_{12}\right)$ describes the motion of the two electrons interacting through the $1 / r_{12} k$ potential in the lack of an external fiezd. Such a method should be valuable for the treatment of the excited states. It has already given promising results for the ground state of $\mathrm{Na}_{2}$ and $\mathrm{K}_{2}$, using three correlated configurations only, but only future work will tell whether the improvement compared to standard configuration interaction techniques is significant.

\section{$5.3-A b$ initio treatment}

Very sophisticated multiconfiguration treatments have been developped for the alkali dimers, especially by Konowalow and coworkers $/ 58,59 /$. It seems at present time that $a b$ initio methods have converged to their optimal result, which, as first demonstrated by Rosmus and Meyer $/ 60 /$, systematically overestimates the equilibrium distance for the ground state potential curve die to a neglect of core polarisation effects.

\section{$5.4-A b$ initio treatment modified for core polarisation effects}

The most impressive progress has been realised in the last two years through the introduction of core-valence correlation effects in the $a b$ initio treatment. In Toulouse $/ 61,62,23 /$ the perturbative treatment of $/ 29 /$ has been reconsidered, and a method proposed in which the valence excitation energy is no more neglected with respect to the core excitation energy. The divergence of the $r^{-4}$ operators is suppressed, and it is no longer necessary to introduce a cut-off radius. The final result for the second order correction is expressed in terms of the core polarisability, the electric field created by the static and transition distributions (a by product of the gradient algorithm in standard molecular programs), the valence excitation energies and a mean energy typical of core excitation. Such a method has provided excellent results for $\mathrm{Na}_{2} / 23 /$ using an empirical value for the core polarisability slightly (7\%) smaller than the experimental one. The experimental curves in the ground state and the first excited states are reproduced within a few wavenumbers accuracy.

We should mention also recent work of Konowalow and Fish on Li2 /24/ in which 
Bardsley's pseudo potential /26/ has been included in an $a b$ initio treatment, neglecting the $V_{3}$ term and hence overestimating the core polarisation effects; composite curves are presented which are intermediate between ab initio calculations 158/ and such results, the two calculations being considered as upper and lower limit for core polarisation corrections.

The results of the four kinds of methods, concerning the ground state of the alkali dimers, are summarised on Table 2, and it is clear that the agreement between theory and experiment is very good.

\section{TABLE 2}

Molecular constants for the ground state of the alkali dimers

\begin{tabular}{|c|c|c|c|c|c|}
\hline & $\operatorname{De}\left(10^{3} \mathrm{~cm}^{-1}\right)$ & $\operatorname{Re}(\AA)$ & $\omega_{e}\left(\mathrm{~cm}^{-1}\right)$ & Method* & Reference \\
\hline \multirow[t]{6}{*}{$\mathrm{Li}_{2}$} & 7.990 & 2.70 & 365.8 & A & $149 /$ \\
\hline & 8.06 & 2.73 & 349 & B & $/ 52 /$ \\
\hline & 8.297 & 2.692 & 347.1 & c & $/ 58 /$ \\
\hline & 8.501 & 2.672 & 350 & D & $/ 24 /$ \\
\hline & 8.5214 & 2.673 & 350 & $\mathrm{E}$ & $163 /$ \\
\hline & 8.5169 & & & $E$ & $/ 21 /$ \\
\hline \multirow[t]{8}{*}{$\mathrm{Na}_{2}$} & 5.72 & 3.04 & 163 & A & $/ 50 /$ \\
\hline & 5.88 & 2.96 & & A & $/ 51 /$ \\
\hline & 6.45 & 3.05 & 165 & B & $/ 52 /$ \\
\hline & 5.64 & 3.07 & 159 & B & $/ 55 /$ \\
\hline & 5.72 & 3.17 & & C & $/ 59 /$ \\
\hline & 6.020 & 3.05 & & D & $/ 23 /$ \\
\hline & 6.024 & & & $E$ & $/ 20 /$ \\
\hline & 6.0226 & & & E & $122 /$ \\
\hline \multirow[t]{5}{*}{$\mathrm{K}_{2}$} & 3.06 & 3.55 & & A & $/ 51 /$ \\
\hline & 5.16 & 3.86 & 99 & $B$ & $152 /$ \\
\hline & 4.27 & 3.85 & 97 & B & $/ 55 /$ \\
\hline & 3.95 & 3.74 & 82 & 0 & 1621 \\
\hline & 4.19 & 3.905 & 92.12 & $E$ & $164 /$ \\
\hline
\end{tabular}

${ }_{A} \quad$ effective potential with configuration interaction

B effective potential with explicit treatment of valence correlation

C $\quad a b$ initio calculations

D $\quad a b$ initio corrected for core-valence correlation effects

E experiment 
CONCLUSION

The intense theoretical activity stimulated by the accuracy of experimental results obtained owing to laser techniques has led to some progress in the description of systems with one and two active electrons.

Concerning alkali-rare gas systems, model potential and pseudo potential techniques, in which the core electrons are simulated by an effective potential, provide a satisfactory description of the physics as far as the ionisation energies are concerned. The rare gas then acts as an additionnal quantum defect. At short internuclear distances, the accurate evaluation of the core-core interaction is still an open problem. The generalisation of the method to excited rare gas-rare gas interactions demands a precise treatment of the core-core term.

Effective potential methods have long been successful in treating alkal i molecular ions. Core polarisation effects are introduced through effective operators which contain a cut-off function to avoid divergence at small $r$. The results are sensitive to the choice of the cut-off radius, and an unambiguous way of determining this parameter, possibly by fitting to photoionisation cross-sections, should be developped.

The generalisation to two active electron systems such as alkali dimers is performed either by configuration interaction techniques or by the use of correlated functions. A comparison of the two approaches for the treatment of excited and Rydberg states will be possible in the next future. The density functionnal formalism has been generalised to the treatment of clusters.

$A B$ initio methods generally provide an overestimation of the bond length, due to a neglect of core polarisation effects. A major improvement has been obtained in the last two years with the introduction of an explicit second order treatment of corevalence correlation in the standard codes. Such a method avoids the difficulties raised by the presence of a cut-off radius in the effective operator method. A spectacular agreement is obtained between theory and experiment for the ground and first excited states of Na2. The comparison of this method with effective operator techniques for the treatment of the highly excited states of the dimers and of the collisional ionisation of the alkalis appears as an appealing prospect for future work.

\section{ACKNOWLEDGEMENTS}

The author wishes to thank Pr. R. McCarroll and Dr. P. Valiron for the many yearscollaboration on the rnodel potential method, and $\mathrm{Pr}$. A. Dalgarno for some enlightening discussions. Contributions of A. Chebanier de Guerra, M. Philippe, M.E. Dolan, D. Hennecart and A.L. Roche to various parts of the work presented here are gratefully acknowledged. Particular thanks are due to A. Henriet, M. Aubert-Frecon and C. Le Sech for the very stimulating collaboration on the alkali dimers problem, and to Pr. P. Pluvinage for his interest in the work. The author is thankful to S. Sandmeier, M. Soyez, B. Chauveau for their help in the preparation of the manuscript.

\section{References}

1. Ahmad Bitar R., Lapatovich W.P., Pritchard D.E., Renhorn I. Phys. Rev. Lett. 39 (1977) 1657

2. Tellinghuisen J., Ragone A., Myung S.Kim, Auerbach D.J., Smalley R.E., Wharton L., Levy D.H. J. Chem. Phys. 71 (1979) 1283

3. Masnou-Seeuws F., Philippe M., Valiron P., Phys. Rev. Lett. 41 (1978) 395

4. Hanssen J., McCarrol] R., Valiron P., J. Phys. B 12 (1979) 899

5. Valiron P., Gayet R., McCarroll R., Masnou-Seeuws F., Philippe M., J. Phys. B 12 (1979) 53

6. Chebanier de Guerra A., Masnou-Seeuws F. Proc. 13th ICPEAC Berl in (1983) 314 and submitted to $\mathrm{J}$. Phys. B.

7. Pascale J. Phys. Rev. A 28 (1983) 632

8. York G., Scheps R., Gallagher A., J. Chem. Phys. 63 (1975) 1052 
9. Mathur B.P., Rothe E.W., Reck G.P., Ligrtman A.J., Cher.. Phys. Lett. 56 (1978) 336

10. Bernheim R.A., Gold L.P., Tipton T. 1. Chem. Phys. 78 (1983) 2625

11. Eisel D., Demtröder W., Chem. Phys. Lett. 88 (1982) 481

12. Eisel D., Demtröder W., Muller W., Botschwina P., Chemical Physics 80 (1983) 329

13. Carlson N.W., Taylor A.J., Schawlow A.L.. Phys. Rev. Lett. 45 (1980) 18

14. Martin S., Chevaleyre J., Bordas C., Valignat S., Broyer M., Cabaud B., Hoareau A. Chem. Phys. Lett. 87 (1982) 235

15. Leutwyler S., Hermann A., Wöste L., Schumacher E. Chemical Physics 48 (1980) 253

16. Broyer M., Chevaleyre J., Delacretaz G., Martin S., Wöste L. Chem. Phys. Lett. 99 (1983) 206

17. Fuentealba P., Preuss H., Stol] H., von Szentpaly L. Chem. Phys. Lett. 89 (1982) 418

18. Henriet A., Masnou-Seeuws, F. Chem. Phys. Lett. 101 (1983) 535

19. Hessel M.M., Vidal C.R. J. Chem. Phys. 70 (1979) 4439

20. Verma K.K., Bahns J.T., Rajaei-Rizi A.R., Stwalley W.C., Zemke W.T. J. Chem. Phys. 76 (1983) 3599

21. Verges J., Bacis R., Barakat P., Carrot P., Churassy S., Crozet P., Chem. Physics Letters 98 (1983) 203

22. Barrow R.F., Vergès J., Effantin C., Hussein K., D'Incan J. ibid 104 (1984) 179

23. Jeung G. H. J. Phys. B 16 (1983) 4289

24. Konowalow D.D., Fish J.L. Chemical Physics 84 (1984) 463

25. Dalgarno A. Atomic Physics vol. 4 (New York Plenum Press) (1975) 325

26. Bardsley J.N., Case studies in atomic physics vol. 4 (Amsterdam North Holland) (1974) 299

27. Veselov M.G., Schtoff A.V. Opt. Spectrosc. 22 (1967) 457

28. Caves T.C., Dalgarno A. J. Quant. Spectr. R.ad. Transf 12 (1972) 1539

29. Bottcher C., Dalgarno A. Proc. R. Soc. A 340 (1974) 187

30. Henriet A., Thèse de 3è cycle (1983) Orsay unpublished

31. Klapisch M. Comput. Phys. Comm. 2 (1971) 239

32. Bottcher C. J. Phys.B 4 (1971) 1140

33. Peach G. J. Phys.B 11 (1978) 2107

34. Valiron P., Roche A.L., Masnou-Seeuws F. and Dolan M.E. J. Phys.B (1984) in press

35. Fermi E. Nuovo Cim. 11 (1934) 157

36. Smirnov B.M. Sov. Phys. JETP 24 (1967) 314

Ivanov 0.K. Opt. Spectrosc.40 (1976) 554

37. Dolan M.E., Masnou-Seeuws F. J. Phys.B 14 (1981) L 583

38. Masnou-Seeuws, F., Boulmer J., Maurin T., Roche A.L., Valiron P., J. Phys.B 15 (1982) 2989

39. Sondergaard N.A., Mason E.A. J. Chem. Phys. 62 (1975) 1299

40. Lamm D.R., Thackston M.G., Eisele F.L., ElT is H.W., Twist J.R., Pope W.P., Gati and I.R., McDaniel E.W. J. Chem. Phys. 74 (1981) 3042

41. Düren R., Tischer H. Chem. Phys. Lett. 79 (1981) 481

42. Düren R., HasseTbrink E., Moritz G. Z. Phys. A 307 (1982) 11

43. Konowalow D.D., Rosenkrantz M.E. Chem. Phys. Lett. 61 (1979) 489

44. Henriet $A$. to be published

45. Weisheit J.A. Phys. Rev. A5 (1972) 1621

46. Bottcher C. J. Phys. B 6 (1973) 2368

47. Colomb de Daunant I., Vassilev G., Baudon J. and Stern B. Journal de Physique 43 (1982) 591

48 Hennecart D., Masnou-Seeuws F. (1984) submitted to J. Phys.B

49. Watson D.K., Cerjan C.J., Guberman S., Dalgarno A. Chem. Phys. Lett. 50 (1977)

50. Bardsley J.N., Junker B.R., Norcross D.W. Cherl. Phys. Lett. 37 (1976) 502

51. Valance A. and Nguyen Tuan Q. J. Phys.B 15 (1982) 17

52. Flad J., Igel G., Dolg M., Stoli H., Preuss P. Chemical Physics 75 (1983) 331

53. Chisholm C.D.M., Öpik O., Proc. Roy. Soc. 83 (1964) 541

54. Norcross D.W. Phys. Rev. Lett. 32 (1973) 192

55. Henriet A., Aubert-Frecon M., Le Sech C., Masnou-Seeuws F. (1984) J. Phys. B in press

56. Pluvinage Ph. Ann. Phys. 5 (1950) 145

Pluvinage Ph. Journal de Physique 12 (1951) 789 
57. Aubert-Frecon M., Le Sech C. J. Chem. Phys. 74 (1981) 2931

58. Konowa low D.D., 01 son M.L. J. Chem. Phys. 71 (1979) 450

59. Konowa 1ow D.D., Rosenkrantz M.E., 01son M.L. J. Chem. Phys. 72 (1980) 2612

60. Rosmus P., Meyer W. J. Chem. Phys. 65 (1976) 492

61. Jeung G.H., MaTrieu J.P., Daudey J.P. J. Chem. Phys. 77 (1982) 3571

62. Jeung G.H., Daudey J.P., Mairieu J.P. J. Phys. B 16 (1983).699

63. Huber K.P., Herzberg G. Constants of Diatomic Molecules (1979) Van Nostrand

64. ibid p. 355 